\title{
Epidemiologia de los casos de COVID - 19 diagnosticados en albergues sanitarios del gran Asunción, Paraguay (2020)
}

\author{
Pasionaria Ramos ${ }^{1,2}$, Elder Silva ${ }^{3}$, Jorge Canese ${ }^{1}$, , Gladys Velázquez ${ }^{1}$ \\ ${ }^{1}$ Universidad Nacional de Asunción, Facultad de Ciencias Médicas, Cátedra de \\ Microbiología. Asunción, Paraguay \\ ${ }^{2}$ Ministerio de Salud Pública y Bienestar Social. MSP y BS. Dirección General de \\ Vigilancia de la Salud (DGVS). Paraguay \\ ${ }^{3}$ Secretaria Municipal de Saúde de Afuá, Pará. Brasil
}

Cómo referenciar este artículo/

How to reference this article:
Ramos P, Silva E, Canese J, Velázquez G. Epidemiologia de los casos de COVID - 19 diagnosticados en albergues sanitarios del gran Asunción, Paraguay (2020). Mem. Inst. Investig. Cienc. Salud. 2021; 19(2): 69-77

\begin{abstract}
RE S U M E N
Paraguay habilitó albergues sanitarios para que guarden cuarentena las personas venidas del exterior, como medida para evitar la propagación de casos de COVI-19. EI objetivo fue describir las características epidemiológicas de los casos de COVID-19 diagnosticados en los albergues sanitarios en el Gran Asunción desde el 7 de marzo al 30 de setiembre. Estudio descriptivo, transversal, temporalmente retrospectivo. El muestreo fue no probabilístico de casos consecutivos. Las variables de los casos confirmados fueron, sexo, edad y distritos. Fueron utilizados datos de acceso abierto del Ministerio de Salud Pública y Bienestar Social. Para análisis estadístico de las variables fueron incluidas frecuencias, expresadas como porcentajes, para lo cual fue utilizado el programa Excel. Se diagnosticaron 390 casos en el Gran Asunción. El mayor número de casos se registró en agosto (118 casos). El 69,2\% (270) de los casos en el Gran Asunción fue del sexo masculino, relación hombre/mujer de 2,3. El

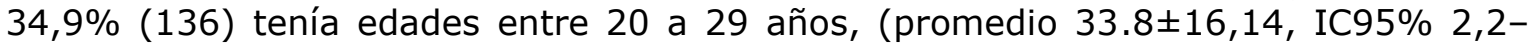
$65,5)$. El $80 \%$ (310) del total se presentó en menores de 50 años. Las ciudades con mayor cantidad de casos fueron Asunción $(46,2 \%, 180)$ y los Distritos de Luque $(8,7 \%, 34)$; Ñemby $(6,4 \%, 25)$ y Capiatá $(5,1 \%, 20)$. Las características epidemiológicas presentadas son las esperadas, dado el perfil sociodemográfico de estas ciudades.
\end{abstract}

Palabras clave: Covid-19, epidemiología, albergues, Paraguay (fuente: DecsBvsalud).

\section{Epidemiology of COVID cases - 19 diagnosed in sanitary shelters in greater Asunción, Paraguay (2020)}

\begin{abstract}
A B S T R A C T
Paraguay opened sanitary shelters to quarantine people arriving from abroad, as a measure to prevent the spread of COVID-19 cases. The objective was to describe the epidemiological characteristics of COVID-19 cases diagnosed in sanitary shelters in Greater Asunción from March 7 to September 30. It was a descriptive, cross-sectional, temporally retrospective study, with non - probabilistic sampling of consecutive cases. The variables of confirmed cases in quarantine facilities were sex, age and
\end{abstract}

Fecha de recepción: Mayo 2021. Fecha de aceptación: Julio 2021

*Autor correspondiente: Gladys Velázquez. Cátedra de Microbiología, Facultad de Ciencias Médicas, Universidad Nacional de Asunción, Rodi 2253 entre Dr. Montero y Guillermo Arias, Asunción, Paraguay

Email: velazquez.aguayo@gmail.com 
departments. Open access data from the Ministry of Public Health and Social Welfare were used. For statistical analysis of the variables, frequencies, expressed as percentages, were included and the Excel program was used for it. Three hundred and ninety cases were diagnosed in Greater Asunción. The highest number of cases was in August (118 cases), 69.2\% (270) of the cases in Greater Asunción were male, with a male/female ratio of $2.3,34.9 \%$ (136) ranged from 20 to 29 years old, (mean 33.8 $\pm 16.14,95 \%$ CI 2.2-65.5). Eighty percent (310) of the cases occurred in the population under 50 years of age. The locations that presented the highest number of cases were Asunción $(46.2 \%, 180)$ and the Districts of Luque $(8.7 \%, 34)$; Nemby $(6.4 \%, 25)$ and Capiatá $(5.1 \%, 20)$. The epidemiological characteristics presented were expected given the socio demographic profile of these cities.

Keywords: COVID-19, epidemiology, sanitary shelters, Paraguay (Source: Decs Bvsalud)

\section{INTRODUCCIÓN}

La Organización Mundial de la Salud (OMS) declaró "emergencia de salud pública" el brote por COVID-19, y finalmente fue declarada "pandemia" el 11 de marzo de $2020^{(1)}$, debido a su rápida diseminación por los distintos países y continentes, comenzando por Asia, epicentro del brote. En Europa, los primeros casos notificados fueron en Alemania, Francia, Italia y España ${ }^{(2,3)}$. En todos los casos se adoptaron las medidas básicas de prevención, precauciones especiales para las visitas y los contactos sospechosos o confirmados de COVID-19 $9^{(4,5)}$ en tanto que, para los profesionales sanitarios, las medidas tomadas fueron aún más estrictas considerando su alto nivel de exposición ${ }^{(4,6-8)}$.

En América del Sur, el primer caso reportado fue en Brasil en el mes de febrero ${ }^{(9)}$, en Paraguay el 7 de marzo ${ }^{(10)}$, y en Uruguay el 13 de marzo ${ }^{(11)}$. Todos los países del Mercado Común del Sur (MERCOSUR) tomaron medidas similares ante la aparición de los casos, evitando así la propagación del brote. La mayoría de las medidas se centraron en la aplicación de la cuarentena y el distanciamiento social ${ }^{(12)}$, el cierre de establecimientos educativos, los eventos públicos, el cierre de centros comerciales, los clubes deportivos, las fronteras y el tráfico aéreo ${ }^{(11)}$.

Aunque las medidas adoptadas por los gobiernos hayan sido similares, cada país presentó algunas particularidades. Así, por ejemplo, en Uruguay, las medidas fueron menos restrictivas que en otros países, ya que no se prohibió la circulación de personas, el funcionamiento de industrias y de comercios pequeños. Estas medidas permitieron mantener el número de contagios relativamente bajo y retrasar el pico de la pandemia(11).

En Paraguay, ante el primer caso diagnosticado, fue establecida la cuarentena educativa y social ${ }^{(13,14)}$, rompiendo de este modo el protocolo de la OMS, que establecía la cuarentena recién en la fase 4, cuando ya existía transmisión comunitaria ${ }^{(15)}$. La primera medida tomada por las autoridades sanitarias fue la suspensión de clases en todos los niveles, básico, secundario y universitario ${ }^{(13,14)}$.

Otra de las medidas tomadas fue la creación de albergues sanitarios en base al decreto No 3526/2020, por el cual se autoriza al Ministerio de Salud Pública y Bienestar Social (MSP y BS) a habilitar albergues destinados para el aislamiento supervisado de personas que hayan dado positivo a los test de COVID a los efectos de hacer frente a la pandemia y evitar su propagación en la población en general ${ }^{(16)}$. Posteriormente, este decreto fue complementado por la Resolución S.G. No 166/2020, la cual establece que las personas que hayan dado positivo a los test de COVID deberán guardar aislamiento supervisado en albergues habilitados para el efecto, y que se encuentren encuadrados en algunos de los siguientes casos: personas que por voluntad propia deseen alejarse de su entorno familiar u hogar; personas en situación de vulnerabilidad que no tengan posibilidad de guardar aislamiento en su hogar; personas que hayan incumplido la medida de aislamiento dispuesta; personas provenientes de países extranjeros que se hallen en cuarentena en lugares dispuestos para el efecto y que hayan dado positivo ${ }^{(17)}$. 
A esta última, se adhirió la Resolución S.G. No 173/2020, la cual establece medidas sanitarias ante el riesgo de expansión del COVID en el territorio nacional. Esta Resolución fue destinada a personas provenientes de otros países, y la misma dispone que paraguayos provenientes de otros países por razones humanitarias deberán cumplir aislamiento preventivo en un albergue u otro lugar designado para el efecto(16). La Resolución S.G. No 212/2020 está referida a los protocolos de orientación para personas que requieran cumplir cuarentena o aislamiento, evaluación y funcionamiento de albergues ${ }^{(18)}$.

En la resolución No $228 / 2020$ de fecha 31 de mayo de 2020 se define como albergue "todo lugar físico que reúna los requerimientos técnicos establecidos por el MSP y BS, para las personas que requieran cumplir cuarentena o aislamiento". Además, define los criterios y funciones de los diferentes estamentos involucrados en el funcionamiento de los albergues ${ }^{(19)}$.

La medida adoptada por el gobierno trajo consigo un gran número de reclamos y quejas, no solamente por el carácter obligatorio del mismo, sino por la infraestructura física y los servicios brindados por los mismos, por lo que posteriormente, fueron creadas las "posadas" y los "hoteles salud", para aquellas personas con voluntad de contar con un mayor confort el tiempo de duración del aislamiento (14 días). Se instalaron 175 albergues, 38 hoteles salud, y 2 posadas salud, dando asistencia a 8.000 personas $^{(20,21)}$.

Asunción es la capital del país y la ciudad más poblada del Paraguay. En el 2020 contaba con 521.559 habitantes $^{(22,23)}$. La dinámica urbana la vincula con la Gran Asunción o el Área Metropolitana de Asunción (AMA), la cual está integrada por la capital y 10 municipios del Departamento Central, representando el $52 \%$ de la población urbana del Paraguay ${ }^{(24)}$ y en donde habita el $74 \%$ de la población metropolitana ${ }^{(25)}$. El objetivo del presente trabajo es describir las características epidemiológicas de los casos de COVID-19 diagnosticados en los albergues sanitarios del Gran Asunción, Paraguay durante los meses de abril a septiembre del año 2020.

\section{METODOLOGÍA}

El estudio fue descriptivo, transversal y temporalmente retrospectivo. El ámbito geográfico considerado fueron las 19 ciudades que componen el Departamento Central, incluyendo la capital, Asunción.

El ámbito temporal considerado fue a partir del día 7 de marzo, fecha del primer caso diagnosticado en el país hasta el 30 de septiembre, ya que los albergues dejaron de funcionar a fines de septiembre aproximadamente ${ }^{(26-8)}$.

El muestreo fue no probabilístico de casos consecutivos. Las variables consideradas fueron: casos confirmados, sexo (masculino, femenino), edad (promedio y desviación estándar) y ciudades del Gran Asunción. En cuanto a la procedencia de los casos, fue considerado el lugar de residencia del caso confirmado de COVID - 19.

Se define como albergue sanitario, "aquellos lugares destinados para el aislamiento supervisado de personas que hayan dado positivo a los test de COVID. Las personas que deberán guardar la cuarentena incluyen a aquellas personas que por voluntad propia deseen alejarse de su entorno familiar u hogar; personas en situación de vulnerabilidad que no tengan posibilidad de guardar aislamiento en su hogar; personas que hayan incumplido la medida de aislamiento dispuesta; personas provenientes de países extranjeros que se hallen en cuarentena en lugares dispuestos para el efecto y que hayan dado positivo" ${ }^{\prime 16,17)}$ y se define como caso confirmado de COVID - 19, "toda persona que presente una prueba RT-PCR positiva para el SARS COV-2"(29).

Como fuente de datos fueron utilizados los datos de acceso abierto disponibles en la página WEB del MSP y $\mathrm{BS}^{(30)}$.

Para análisis estadístico de las variables fueron incluidas frecuencias, expresadas como porcentajes, para lo cual fue utilizado el programa Excel.

En cuanto a los criterios éticos, al tratarse de una investigación con fuente de datos secundarios sin participación de seres humanos, el protocolo del presente trabajo no requirió la aprobación del Comité de Ética en Investigación. 


\section{RESULTADOS}

Entre marzo y septiembre del 2020 han sido diagnosticados390 casos de COVID-19 en los albergues sanitarios. En cuanto a la procedencia de los casos, 53,8\% (210/390) se presentaron en el Departamento Central y el 46,2\% (180/390) en Asunción. El mes con menor número de casos positivos fue el mes de abril, con 14 casos, y el mes con mayor número de casos fue el mes de agosto con 118 casos (Figura 1).

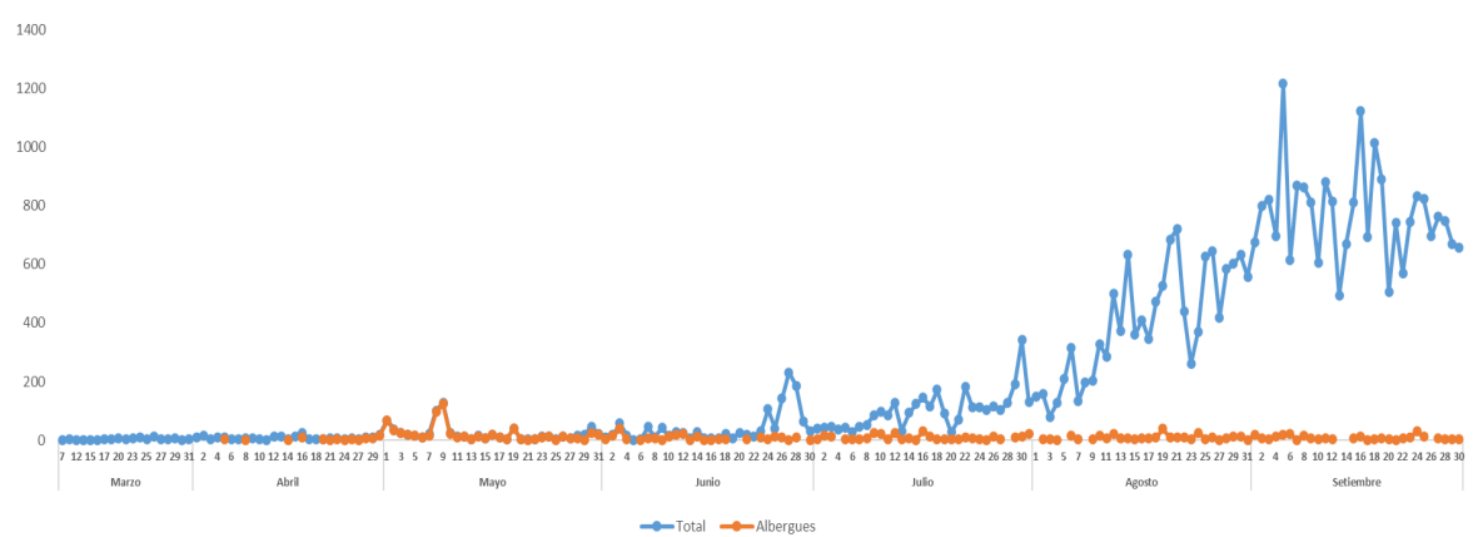

Figura 1. Distribución de casos de COVID-19 diagnosticados en albergues sanitarios del Gran Asunción, Paraguay. (Abril-Setiembre. 2020). N: 390

Fuente: Ramos P, Elder Silva E., Canese J, Velázquez G. Epidemiologia de los casos de COVID - 19 diagnosticados en albergues sanitarios del Gran Asunción, Paraguay (2020)

El 69,2\% (270/390) de los casos fueron del sexo masculino, con una razón hombre/mujer de 2,3. El promedio de edad fue de 33,8 años, \pm de 16,14 (IC95\% 2,2$65,5)$. El 34,5\% de los casos (136/390) tenía edades comprendidas entre 20 a 29 años. El 80\% (310/390) de los casos confirmados fue en menores de 50 años. Al relacionar el sexo con la edad, la mayor cantidad de casos en el sexo masculino se presentó en el grupo de edad de 20 a 29 años (34,5\%, 136/390). En tanto, el 4,1\% $(16 / 390)$ de los casos registrados en la población femenina, se presentaron en las menores de10 años (Tablas 1 y 2, Figura 2).

Tabla 1. Características demográficas de los casos de COVID 19 en albergues temporales en el Gran Asunción, Paraguay (abril - septiembre 2020). N: 390

\begin{tabular}{lrr}
\hline Variables Demográficas & \multicolumn{2}{c}{ Frecuencia } \\
\cline { 2 - 3 } Sexo & $\mathbf{N}$ & $\mathbf{\%}$ \\
\hline Masculino & 270 & 69,2 \\
Femenino & 120 & 30,8 \\
Total de Casos & $\mathbf{3 9 0}$ & $\mathbf{1 0 0}$ \\
$<10$ & 16 & 4,1 \\
$10-19$ & 44 & 11,3 \\
$20-29$ & 136 & 34,5 \\
$30-39$ & 65 & 16,7 \\
$40-49$ & 49 & 12,6 \\
$50-59$ & 51 & 13,1 \\
$60-69$ & 21 & 5,4 \\
$70-79$ & 7 & 1,8 \\
$>80$ & 1 & 0,3 \\
Total de Casos & $\mathbf{3 9 0}$ & $\mathbf{1 0 0}$ \\
\hline
\end{tabular}

Fuente: Ramos P, Elder Silva E., Canese J, Velázquez G. Epidemiologia de los casos de COVID 19 diagnosticados en albergues sanitarios del Gran Asunción, Paraguay (2020) 


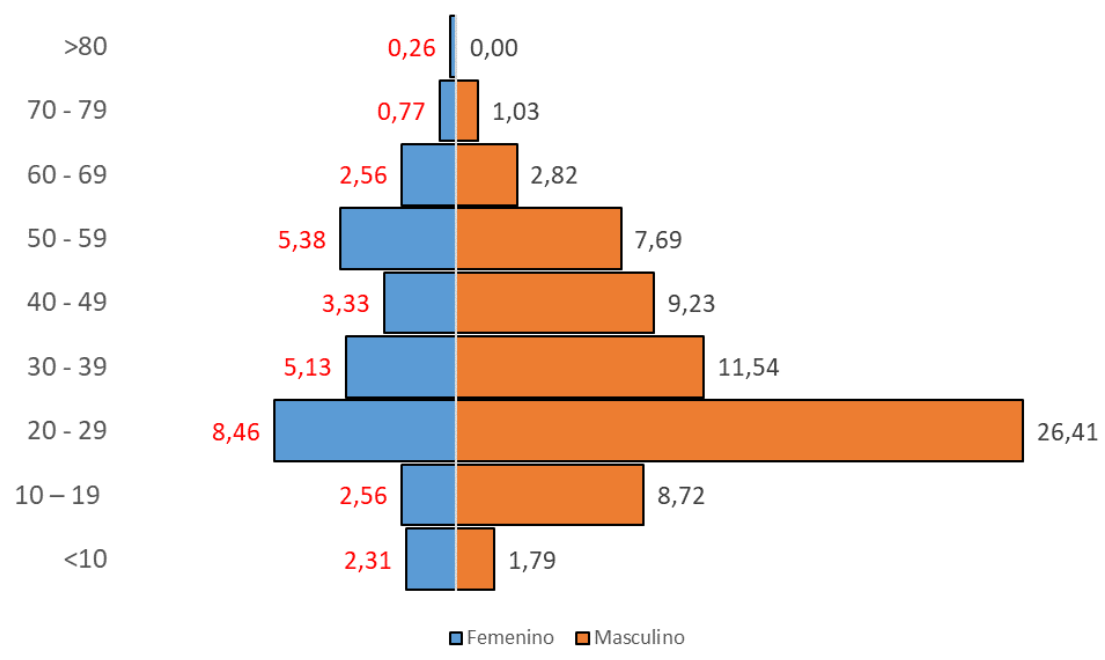

Figura 2. Pirámide porcentual de los casos de COVID 19 en albergues sanitarios en el Gran Asunción, Paraguay (abril - septiembre 2020). N: 390

En cuanto a la distribución geográfica de los casos según los Distritos que componen el Gran Asunción, los distritos con mayor cantidad porcentual de casos se presentaron en la capital Asunción $(46,2 \%, 180)$ y los Distritos de Luque $(8,7 \%, 34)$; Ñemby $(6,4 \%, 25)$ y Capiatá $(5,1 \%, 20)$.

Al relacionar la distribución de los casos de COVID entre las ciudades y el sexo, en el Gran Asunción, la mayoría de los casos masculinos se presentaron en los distritos de Villeta, Ypacaraí e Itauguá. En tanto, la mayor cantidad de casos femeninos se presentó en los distritos de Nueva Italia, Guarambaré y Ñemby. No se presentaron casos femeninos en los distritos de Villeta e Ypacaraí (Tabla 2).

Tabla 2. Distribución de los casos de COVID 19 en albergues sanitarios por sexo y lugar de procedencia en el Gran Asunción, Paraguay (abril - septiembre 2020). N: 390

\begin{tabular}{|c|c|c|c|c|c|}
\hline \multirow{2}{*}{$\begin{array}{l}\text { Asunción y distritos del } \\
\text { Gran Asunción }\end{array}$} & \multicolumn{2}{|c|}{ Masculino } & \multicolumn{2}{|c|}{ Femenino } & \multirow[b]{2}{*}{ Tota } \\
\hline & $\mathbf{N}$ & $\%$ & $\mathbf{N}$ & $\%$ & \\
\hline Asunción & 124 & 68,9 & 56 & 31,1 & 180 \\
\hline Luque & 28 & 82,4 & 6 & 17,6 & 34 \\
\hline Ñemby & 10 & 40 & 15 & 60 & 25 \\
\hline Capiatá & 14 & 70 & 6 & 30 & 20 \\
\hline Lambaré & 13 & 68,4 & 6 & 31,6 & 19 \\
\hline San Lorenzo & 13 & 72,2 & 5 & 27,8 & 18 \\
\hline Itauguá & 15 & 93,8 & 1 & 6,3 & 16 \\
\hline Fernando de la Mora & 6 & 42,9 & 8 & 57,1 & 14 \\
\hline M. R. Alonso & 9 & 81,8 & 2 & 18,2 & 11 \\
\hline Guarambaré & 3 & 33,3 & 6 & 66,7 & 9 \\
\hline Itá & 7 & 77,8 & 2 & 22,2 & 9 \\
\hline Ypané & 7 & 87,5 & 1 & 12,5 & 8 \\
\hline Limpio & 6 & 85,7 & 1 & 14,3 & 7 \\
\hline San Antonio & 6 & 85,7 & 1 & 14,3 & 7 \\
\hline Areguá & 3 & 75 & 1 & 25 & 4 \\
\hline J. A Saldívar & 2 & 66,7 & 1 & 33,3 & 3 \\
\hline Villa Elisa & 1 & 50 & 1 & 50 & 2 \\
\hline Ypacaraí & 2 & 100 & 0 & 0 & 2 \\
\hline Nueva Italia & 0 & 0 & 1 & 100 & 1 \\
\hline Villeta & 1 & 100 & 0 & 0 & 1 \\
\hline \multicolumn{6}{|l|}{ Central } \\
\hline Total & 270 & 69,2 & 120 & 30,8 & 390 \\
\hline
\end{tabular}

Fuente: Ramos P, Elder Silva E., Canese J, Velázquez G. Epidemiologia de los casos de COVID 19 diagnosticados en albergues sanitarios del Gran Asunción, Paraguay (2020) 


\section{DISCUSIÓN}

Al inicio de la pandemia, Paraguay constituyó uno de los países con menor número de casos de América del Sur, luego de Venezuela y Uruguay, y en comparación con Estados Unidos de América (30.628.805 casos), Brasil (12.910.082casos) y Colombia $(2.428 .048)^{(31)}$.

A mediados de 2020, Paraguay presentaba una letalidad muy baja, $(0,95)$, comparada con países como Inglaterra, $(15,5)$, México $(12,2)$, Estados Unidos $(5,1)$, y Brasil $(3,1)^{(31)}$. Esto podría explicarse por las rápidas medidas tomadas tanto por el gobierno nacional como por el Ministerio de Salud, siendo el primero en habilitar los albergues temporales exclusivos para guardar cuarentena y evitar así la rápida diseminación del virus ${ }^{(15,17-22)}$.

Sin embargo, actualmente, presenta tasas de letalidad media de 2,0 semejantes a Portugal y Francia, pero menores y cercanas con países como Brasil $(2,5)$, Argentina $(2,4)$, Chile $(2,3)^{(31)}$. La mortalidad en los últimos 14 días, con fecha de 23 de marzo, ha sido de 3,9 por 100.000 habitantes, con una letalidad de $1,3 \%{ }^{(32)}$.

El primer caso de COVID-19 fue confirmado el día 7 de marzo. Y a partir del 4 de abril hasta el 30 de septiembre del 2020 han sido diagnosticados 1.581 casos en los Albergues y Hoteles Salud de todo el país. El 39\% (861) de los casos fue diagnosticado en los albergues sanitarios, ya que, por protocolo, en un primer momento, las pruebas eran realizadas al término de los 14 días; posteriormente el test fue realizado al ingresar al albergue ${ }^{(33,34)}$.

La mayoría de los casos de COVID-19 se concentraron en Asunción y el Departamento Central con 390 casos. El 53,8\% (210) de los casos pertenecía al Departamento Central y el 46,2\% (180) a Asunción. El mes con mayor número de casos fue el mes de agosto con 118 casos y el mes con menor número fue el mes de abril, con 14 casos. Asunción, la capital del país, fue la ciudad donde fueron confirmados los primeros casos, por lo tanto, era de esperar una mayor cantidad de los mismos. De la misma manera y en el mismo periodo en estudio, ciudades como Madrid, París y Buenos Aires presentaron una alta tasa de incidencia de casos, mientras que Roma, Berlín y Milán, lo hicieron en menor cantidad, teniendo en cuenta su alta densidad poblacional. Esta diferencia podría deberse a las diferentes estrategias utilizadas por estas ciudades para contener el virus ${ }^{(35)}$. Con relación a América del Sur, las ciudades más afectadas fueron San Paulo, Rio de Janeiro, Santiago, Ciudad de México y Lima ${ }^{(36)}$.

El 69,2 \% de los casos fue del sexo masculino, predominio presentado en ciudades como Wuhan, con un $68 \%$, Ciudad de México56\%, entre otras ${ }^{(37,38)}$. En ciudades como Teherán la relación hombre/mujer se presentó con 1,7 cercanas a la presentada en el presente artículo con $2,3^{(39)}$. La alta prevalencia del sexo masculino puede justificarse por el hecho de que los hombres no hayan cumplido correctamente las medidas de restricción, debido al apremio para mantener el sustento del hogar, aumentando así la exposición ${ }^{(34)}$.

El promedio de edad fue de 33,8 años, El 34,9\% de los casos (136) tenía edades comprendidas entre 20 a 29 años. El $80 \%$ (310) de los casos confirmados fue en menores de 50 años. Esto se debería a la pirámide poblacional. Paraguay cuenta con 1.954.150 jóvenes de 15 a 29 años, y 486.662 adultos mayores (65 años y más) ${ }^{(40)}$.

A la hora de revisar la literatura referida al COVID-19 no se conoce otros países que hayan creado los albergues sanitarios del modo como fueron implementados en Paraguay. La bibliografía refiere investigaciones realizadas en albergues, entendiendo estos lugares, como lugares de acogida para las personas sin hogar, de ahí la importancia que esta pequeña investigación podría aportar.

\section{CONCLUSIÓN}

Las características epidemiológicas presentadas en los casos de albergues sanitarios del Gran Asunción y Asunción son las esperadas dado el perfil sociodemográfico de estas ciudades.

Financiamiento: no se ha recibido ningún financiamiento para la realización de esta investigación. 
Conflicto de interés: los autores declaran no tener ningún conflicto de interés.

Contribución de los autores: Todos los autores han contribuido de igual manera para la investigación y la redacción del presente trabajo.

\section{REFERENCIAS BIBLIOGRÁFICAS}

1. Organización Panamericana de la Salud (OPS)/OMS Brasil. La OMS afirma que COVID-19 ahora se caracteriza como una pandemia. [Internet]. Ginebra: WHO; 2020 [citado el 27 de marzo de 2020]. Disponible en:

https://www.paho.org/bra/index.php?o ption $=$ com content $\&$ view $=$ article\&id $=6$ 120:oms-afirma que-covid-19-e-agoracaracterizada-como-

pandemia\&Itemid $=812$

2. Giovanetti M, Benvenuto D, Angeletti S, Ciccozzi M. The first two cases of 2019 nCoV in Italy: Where they come from? J Med Virol. 2020; 92(5):518-21. doi. 10.1002/jmv.25699

3. Peña-Otero $D$, Díaz-Pérez $D$, de-laRosa-Carrillo D, Bello-Dronda S. ¿Preparados para el nuevo coronavirus? Arch Bronconeumol. 2020; 56(4): 1956. DOI: $10.1016 /$ j.arbres.2020.02.009

4. World Health Organization. Advice on the use of masks in the community during home care and in health care settings in the context of the novel coronavirus (2019-nCoV) outbreak. Interim guidance. [Internet]. Ginebra: WHO; 7 de abril 2020 [citado el 12 de febrero de 2020] Disponible en:

https://apps.who.int/iris/bitstream/han dle/10665/331493/WHO-2019-nCoVIPC Masks-2020.2-eng.pdf

5. Centers for Disease Control and Prevention (CDC). COVID-19. Interim recommendations for Emergency Medical Services (EMS) systems and 911 public safety answering points (PSAPs) for 2019-n CoV in the United States. [Internet]. Atlanta: CDC; 18 de Julio 2020 [citado el 15 de Julio de 2020]. Disponible en:

https://www.cdc.gov/coronavirus/2019ncov/hcp/guidance-for-ems.html

6. Ministerio de Sanidad. Gobierno de España. Información Científica-Técnica. Nuevo coronavirus 2019-nCoV. Instituto de Salud Carlos III. [Internet]. Madrid: Secretaria de Estado de Sanidad. Dirección General de Salud Pública, Calidad e Innovación. Centro de Coordinación de Alertas y Emergencias Sanitarias; 2020 [citado 12 de febrero de 2020]. Disponible en:

https://www.mscbs.gob.es/profesionale s/saludPublica/ccayes/alertasActual/nCo v/documentos/ITCoronavirus.pdf

7. World Health Organization. Infection, prevention and control during health care when novel coronavirus (nCoV) infection is suspected: Interim guidance. [Internet]. Ginebra: WHO; 25 de enero 2020 [citado el 5 de octubre de 2020]. Disponible en:

https://apps.who.int/iris/handle/10665/ 330674

8. Europe Center Disease Prevention and Control. Weekly surveillance report on COVID-19 [Internet]. Solna: ECDC; 1 de Julio 2020 [citado el 7 de enero de 2020]. Disponible en:

https://www.ecdc.europa.eu/en/covid19/surveillance/weekly-surveillancereport

9. Brasil. Ministério da Saúde. Secretaria de Vigilância em Saúde. Centro de Operações em Emergências em Saúde Pública - COVID-19. Boletim Epidemiológico 06. Doença pelo Coronavírus. Brasilia: COE-COVID 19 [Internet] 1 de Julio 2020 [citado el 3 de abril de 2020]. Disponible en: https://portalarquivos.saude.gov.br/ima ges/pdf/2020/April/03/BE6-BoletimEspecial-do-COE.pdfl

10. Paraguay. Ministerio de Salud Pública y Bienestar Social. Dirección General de Vigilancia de la Salud. Asunción: DNVS. [Internet] 1 de Julio 2020 [citado el 31 de marzo de 2020]. Disponible en: https://www.mspbs.gov.py/dependenci as/portal/adjunto/48b432-

Tablero31.03.pdf.

11. Bastos F, Feal-Zubimendi, S, Hernaiz D, Miller SJ, Queijo von Heideken V, Rossi $\mathrm{JL}$, et al. Washington D.C: BID. Departamento de Países del Cono Sur (CSC). ALC Post COVID-19: Retos y oportunidades para países del Cono Sur. 2020. [Internet]. [citado el 20 de agosto de 2020 Disponible en: $10.18235 / 0002372$

12. Center for Disease Control and Prevention (CDC). Implementation of mitigation strategies for communities with local COVID-19 transmission [Internet]. Washington, DC: Center for Disease Control and Prevention; 2019 [citado el 27 de marzo de 2020]. Disponible en:

https://www.cdc.gov/coronavirus/2019ncov/downloads/community-mitigationstrategy.pdf

13. Paraguay. Ministerio de Salud Pública y Bienestar Social. COVID-19: La salud y la vida como bandera. [Internet]. Asunción: MSP y BS; 2020. [citado el 1 de Julio de 2020]. Disponible en: https://www.mspbs.gov.py/portal/2122 
4/covid-19-la-salud-y-la-vida-comobandera.html

14. Paraguay. Poder Ejecutivo. Ministerio de Salud Pública y Bienestar Social. Resolución S. G. n 90 . Por la cual se establecen medidas para mitigar la propagación del coronavirus (COVID19). [Internet]. Asunción: PE; 2020 [Citado 10 de marzo de 2020 Mar 10]. Disponible en:

https://www.mspbs.gov.py/dependenci as/portal/adjunto/5a7857RESOLUCION SG90COVID19.pdf

15. Paraguay. Ministerio de Salud Pública y Bienestar Social. Decreto $n^{\circ}$ $3526 / 2020$. Por el cual se autoriza al Ministerio de Salud Pública y Bienestar Social a habilitar albergues destinados para el aislamiento supervisado de personas que han dado positivo en los test de corona virus (COVID-19). [Internet]. Asunción: MSP y BS; 2020. [citado el 9 de abril de 2020]. Disponible en:

https://www.mspbs.gov.py/dependenci as/portal/adjunto/1c4cb9-

DECRETO3526.PDF

16. Paraguay. Ministerio de Salud Pública y Bienestar Social. Resolución SG No 173/2020. [Internet]. Asunción: MSP y BS; 2020. [citado el 16 de abril de 2020]. Disponible en:

https://www.mspbs.gov.py/dependenci as/portal/adjunto/5f31c2-

ResolucinN173Medidasdestinadasaperso nasprovenientesdeotrospases.pdf

17. Paraguay. Ministerio de Salud Pública y Bienestar Social. Resolución SG No. 166/2020. [Internet]. Asunción: MSP y BS; 2020. [citado el 11 de abril de 2020]. Disponible en:

https://www.mspbs.gov.py/dependenci as/portal//1c8572-

RESOLUCIONSG.N166.pdf

18. Resolución S.G. No 212/2020. Ministerio de Salud Pública y Bienestar Social. [Internet]. Asunción: MSP y BS; 2020. [citado el 9 de mayo de 2020]. Disponible en:

https://www.mspbs.gov.py/dependenci as/portal/adjunto/325e8f-

RESOLUCIONSG.N212.pdf

19. Paraguay. Ministerio de Salud Pública y Bienestar Social. Resolución S.G. N $228 / 2020$. Por la cual se aprueba un nuevo protocolo de orientación para personas que requieran cumplir cuarentena o aislamiento, evaluación y funcionamiento de albergues, en el marco del estado de emergencia sanitaria declarado por el gobierno de la república en la lucha contra la pandemia de coronavirus (COVID-19); y se abroga la Resolución S.E. N 212, de fecha 9 de mayo de 2020. [Internet]. Asunción: MSP y BS; 2020 [citado 31 de mayo de 2020]. Disponible en:

https://www.mspbs.gov.py/dependenci as/portal/adjunto/82b3a5-

RES228DE2020.pdf

20. Rodríguez A. Albergues en Paraguay: la trinchera contra el COVID-19 [Internet]. Buenos Aires: Infobae; 2020 [Citado 3 de Julio de 2020]. Disponible en: https://www.infobae.com/america/agen cias/2020/07/03/albergues-en-

paraguay-la-trinchera-contra-el-covid19/

21. Paraguay. Secretaria Nacional de Turismo. Instalaciones Hoteleras. Estrategias Hotel Salud. [Internet]. Asunción: SNT; 2020 citado el 15 de setiembre de 2020]. Disponible en: https://www.senatur.gov.py/materiales /archivos/0700_15_09_2020.pdf

22. Paraguay. Ministerio de Salud Pública y Bienestar Social. COVID-19: Isolation in quarantine facilities, a key strategy in containing the pandemic. [Internet]. Asunción: MSP y BS 2020 [citado 31 de Julio de 2020]. Disponible en: https://www.mspbs.gov.py/portal/2141 2/covid-19-aislamiento-en-alberguesuna-estrategia-clave-en-la-contencionde-la-pandemia.html

23. Paraguay. Dirección General de Estadística, Encuesta y Censos Proyección de la población por sexo y edad, según Distrito, 2000-2025. [Internet]. Fernando de la Mora; DGEEC; 2015. Disponible en: https://web.archive.org/web/20151016 011537/

http://www.dgeec.gov.py/Publicaciones $\angle$ Biblioteca/proyeccion\%20nacional/Proy eccion\%20Distrital.pdf

24. Paraguay. Atlas censal del Paraguay. [Internet]. Fernando de la Mora: DGEEC.2002. Disponible en:

http://www.bibliotecaroosevelt.edu.py/c gi-bin/koha/opac-

detail.pl?biblionumber $=6209$

25. Meza P. Uso de los Sistemas Globales BREEAM-CM y LEED-ND en Asunción, Paraguay. Use of the BREEAM-CM and LEED-ND Global System in Asunción, Paraguay. Territorios en formación. 2019; 15:171-190. 10.20868/tf.2019.15.4006

26. Diario El Nacional. Ejecutivo debe decidir sobre cierre de 76 albergues para cuarentena. Disponible en: https://www.elnacional.com.py/politica/ 2020/09/23/ trashed-9/. Accesado: 03 de diciembre del 2020.

27. Ministerio de Relaciones Exteriores. Disponible: 
https://www.mre.gov.py/index.php/noti cias-de-embajadas-y-consulados/elgobierno nacional-reivindica-losalbergues-sanitarios-y-rechaza-lasdenuncias-de-amnistia-internacional. Accesado: 03 de diciembre del 2020.

28. Diario HOY. Disminuyen casos provenientes del exterior y albergues podrían dejar de funcionar. Disponible en:

https://www.hoy.com.py/nacionales/co vid-19-disminuyen-casos-provenientesdel-exterior-y-albergues-podrian-dejarde-funcionar.

Accesado: 03 de diciembre del 2020.

29. Paraguay. Ministerio de Salud Pública y Bienestar Social. COVID-19. Protocolo para la Vigilancia Epidemiológica y manejo del aislamiento preventivo. Disponible:

https://www.mspbs.gov.py/dependenci as/portal/adjunto/c1c79a-

ProtocoloVigilanciaEpidemiolgica.pdf.

Accesado: 22 de julio del 2021

30. Paraguay. Ministerio de Salud Pública y Bienestar Social. COVID-19: Reporte Semanal [Internet]. Asunción: Ministerio de Salud Pública y Bienestar Social. Dirección General de Vigilancia Sanitaria. 2020 [citado 30 de noviembre de 2020]. Disponible en: https://public.tableau.com/profile/mspb s\#!/vizhome/COVID19PYReporteSeman al/Covid19R Semanal

31. Paraguay. Ministerio de Salud Pública y Bienestar Social. COVID-19: Registro Confirmado [Internet]. Asunción: Ministerio de Salud Pública y Bienestar Social. Dirección General de Vigilancia Sanitaria. 2020 [citado el 28 de noviembre de 2020]. Disponible en: https://public.tableau.com/profile/mspb s\#!/vizhome/COVID19PY-

Registros/Descargardatos

32. John Hopkins University and Medicine. Coronavirus Resource Center. Global Map. COVID-19 Dashboard by the Center for Systems Science and Engineering (CSSE) at John Hopkins University (JHU). [citado el 5 de octubre de 2020]. Disponible en:

https://coronavirus.jhu.edu/map.html

33. OPS/OMS Paraguay. COVID-19. Reporte de situación No. 47. 2021. [Internet]. Asunción: OPS/OMS. [citado 13 de marzo de 2021]. Semana Epidemiológica (SE) 10. Disponible en: https://www.paho.org/es/documentos/c ovid-19-reporte-situacion-47-paraguay13-marzo-2021

34. Paraguay. Ministerio de Salud Pública y Bienestar Social. Ingresados a albergues son sometidos a test de COVID 19. [Internet] Asunción; MSP y
BS: 2020 [citado el 7 de mayo de 2020]. Disponible en:

https://www.mspbs.gov.py/portal/2089 5/ingresados-a-albergues-sonsometidos-a-test-de-covid-19.html

35. Ramos $P$, Velázquez $G$, Silva $E$, Centurión C, Dullak R, Armoa L. Epidemiologia De Los Casos De Covid 19 Diagnosticados En Albergues Temporales Y Hoteles "Salud", Paraguay (2020). [Internet] Madrid; Jornadas Iberoamericanas Covid. Disponible en: https://www.easp.es/web/jornadasibero americanascovid/comunicaciones/modul es/request.php?module $=0 C$ _proceeding s\&action=view.php\&id=117\&file=1/117. pdf\&a=Accept

36. Gutierrez I. La pandemia en las grandes ciudades del mundo: hasta 10 veces menos contagios que en Madrid, parques abiertos y más restricciones. [Internet] Madrid; El diario.es [citado el 30 de setiembre de 2020]. Disponible en:

https://www.eldiario.es/internacional/p andemia-grandes-ciudades-mundo-10veces-contagios-madrid-parquesabiertos-restricciones 1 6255830.html

37. LA Network. COVID-19 en Latinoamérica: las 30 ciudades con más víctimas por la pandemia. [Internet]: Medellín: LA Network. 2020 [citado el 23 de julio de 2020]. Disponible en: https://la.network/covid-19-en-

latinoamerica-las-30-ciudades-con-masvictimas-por-la-pandemia/

38. Chen N, Zhou M, Dong X, Qu J, Gong F, Han $Y$, et al. Epidemiological and clinical characteristics of 99 cases of 2019 novel coronavirus pneumonia in Wuhan, China: a descriptive study. The Lancet, 2020; 395: 507-513. 10.1016/S01406736(20)30211-7.

39. Secretaría de Salud. Datos Abiertos Dirección General de Epidemiología. [Internet] Ciudad de México: DGE; 2020 [actualizado al 24 de junio de 2020]. Disponible en: https://buff.ly/38rFyRu.

40. Nematollahi S, Shariatpanahi M, Hosseini R, Fatemi A. Why are Men more susceptible to COVID-19: A narrative review of current global knowledge. Men's Health Journal. 2020; 4(1): e1. Disponible en: https://buff.ly/3dX9UMN.

41. Paraguay. Dirección General de Estadística (DGEE). Encuestas y Censos. Paraguay; 2020 [Internet] Asunción: DGEE: 2020. [citado el 10 de mayo de 2020]. Disponible en: https://www.dgeec.gov.py/default.php? publicacion $=33$ 